\title{
MITOCHONDRIAL GENETIC HAPLOGROUPS AND INCIDENT OBESITY: A LONGITUDINAL COHORT STUDY
}

Running title: mithochondrial DNA and obesity

Nicola Veronese* a,b, MD, Brendon Stubbs ${ }^{\mathrm{c}}, \mathrm{PhD}, \mathrm{Ai}_{\text {Koyanagi }}^{\mathrm{d}}, \mathrm{MD}$, Alberto Vaona ${ }^{\mathrm{e}}, \mathrm{PhD}$, Jacopo Demurtas ${ }^{\mathrm{f}}, \mathrm{MD}$, Patricia Schofield ${ }^{\mathrm{g}}, \mathrm{MD}$, Trevor Thompson ${ }^{\mathrm{h}}, \mathrm{PhD}$, Stefania Maggi ${ }^{\mathrm{a}}$, MD

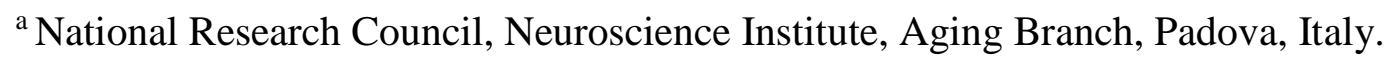

b Ambulatory of Nutrition, IRCCS "S. de Bellis", National Institute of GastroenterologyResearch Hospital, Castellana Grotte, Bari, Italy.

${ }^{c}$ South London and Maudsley NHS Foundation Trust, Denmark Hill, London SE5 8AZ, United Kingdom; Institute of Psychiatry, Psychology and Neuroscience (IoPPN), King's College London, De Crespigny Park, London SE5 8 AF, United Kingdom; Faculty of Health, Social Care and Education, Anglia Ruskin University, Chelmsford, United Kingdom.

${ }^{\mathrm{d}}$ Research and Development Unit, Parc Sanitari Sant Joan de Déu, Fundació Sant Joan de Déu, CIBERSAM, Sant Boi de Llobregat, Barcelona, Spain.

${ }^{\text {e }}$ Primary Care Department, Azienda ULSS20 Verona, Verona, Italy.

${ }^{\mathrm{f}}$ Primary Care Department, Azienda USL Toscana Sud Est, Grosseto, Italy.

g Faculty of Health, Social Care and Education, Anglia Ruskin University, Chelmsford, United Kingdom.

${ }^{\mathrm{h}}$ Faculty of Education and Health, University of Greenwich, London, UK.

*corresponding author. Nicola Veronese +39 0498211776, ilmannato@ gmail.com 


\begin{abstract}
Background/Objectives: A small number of case-control studies have suggested that mitochondrial haplogroups could be associated with obesity. We examined whether obesity risk was influenced by mitochondrial haplogroup in a large North American cohort across an 8 year period. We conducted a longitudinal cohort study including individuals from the Osteoarthritis Initiative.

Subjects/Methods: Mitochondrial haplogroups were determined by sequencing and PCRRFLP techniques using this nomenclature: HV, JT, KU, IWX, and superHV/others. The strength of the association between mitochondrial haplogroups and incident obesity was quantified with hazard ratios (HRs) adjusted for potential confounders using Cox's regression. Results: Overall, 2,342 non-obese Caucasian participants (56.7\% women) with a mean \pm SD age of $62.0 \pm 9.5$ years at baseline were included. During a median follow-up of 8 years, 334 individuals $(=14.3 \%$ of baseline population) became obese. After adjusting for 9 potential confounders the haplogroups IWX carried a significant $48 \%$ higher risk of obesity $(\mathrm{HR}=1.48$; 95\%CI: 1.02-2.39) compared to the HV haplotype (the most frequent type).

Conclusion: Only the presence of the IWX haplogroups appears to be linked to increased obesity risk, independent of potential baseline confounders. Future cohort studies are needed with independent data are needed to confirm these findings and to determine potential underlying mechanisms.
\end{abstract}

Keywords: mitochondrial haplogroups; obesity; BMI; Osteoarthritis Initiative. 


\section{INTRODUCTION}

The mitochondrial genome is a circular set of about 17,000 base pairs encoding 37 genes in human beings. Of them, 13 are translated in proteins that are involved in a diverse range of processes relevant to cellular function and survival, e.g. electron transport and oxidative phosphorylation. ${ }^{1}$ Mitochondrial DNA (mtDNA) often undergoes pathological changes and is reported that repair mechanisms are less efficient than nuclear DNA. ${ }^{1,2}$ In addition, the mtDNA evolves much more quickly than average nuclear DNA, with mutations therefore accumulating sequentially along radiating maternal lineages. ${ }^{3}$ Mitochondrial haplogroups are defined as specific groups of single nucleotide polymorphisms (SNPs) which result from mismatches, and it has been increasingly recognized that different mitochondrial haplogroups may influence propensity to several chronic disease states. ${ }^{4-6}$

Mitochondria are essential in energy metabolism, and thus mitochondrial dysfunction could affect obesity. ${ }^{7}$ However, the literature regarding the possible association between mitochondrial haplogroups and obesity is still limited to a small number of case-control studies. In 2,286 Caucasians of Northern European origin living in the USA, Yang et al. found that haplogroup $\mathrm{X}, \mathrm{mt} 4823$ and $\mathrm{mt} 8873$ were related to lower body mass index (BMI) and decreased body fat mass. ${ }^{8}$ Similarly, in an Italian study including 500 obese cases vs. 216 controls, obese people demonstrated greater frequency of haplogroup $\mathrm{T}$ and reduced frequency of haplogroup $\mathrm{J}$ compared to healthy controls. ${ }^{9}$ Finally, in another more recent case-control study involving 1,251 obese people vs. 861 normal weight individuals, haplogroup $\mathrm{T}$ was found to be more frequent in obese people. ${ }^{10}$

Whilst these studies have advanced our knowledge regarding the possible association between mitochondrial haplogroups and obesity, a number of limitations persists. First, they all rely on 
a case-control study design, thus their nature does not allow the authors to infer a cause-effect direction. Second, these studies did not control for any possible confounders (e.g. physical activity or educational level or the presence of other co-morbidities), and it is possible that residual confounding exists.

As little or no longitudinal research has investigated the influence of mitochondrial haplogroups on incident obesity, and some previous case-control studies have reported insignificant associations between mitochondrial haplogroups and obesity ${ }^{11}$, we sought to determine whether obesity risk was linked to different mitochondrial haplogroups in a large prospective cohort of North American people. 


\section{METHODS}

\section{Data source and participants}

Data used in this study were obtained from the Osteoarthritis Initiative (OAI) (http://www.oai.ucsf.edu), a large ongoing nationwide study sponsored by the National Institute of Health. Data was based on patients recruited between February 2004 and May 2006 from the following four clinical sites in the US with a high risk of knee osteoarthritis. Specific data used in the current study were: baseline and screening evaluations (V00; November 2008), and assessments until the final (96 month) available evaluation (V01, V03, V05, V06, V08, V10). Written informed consent provided by all subjects.

\section{Exposure}

Haplogroup assignment was determined by PCR-RFLP and sequencing methods using established methods ${ }^{12}$. The latter consisted of the multiplex assignment of the six main SNPs that contribute to the generation of the most prevalent Caucasian haplogroups ${ }^{13}(\mathrm{H}, \mathrm{V}$, super $\mathrm{HV}, \mathrm{U}, \mathrm{K}, \mathrm{T}, \mathrm{J})$ following the single base extension (SBE) assay.

In line with OAI nomenclature, haplogroups were as follows: H, U, K, J, T, V, SuperHV, I, $\mathrm{W}, \mathrm{X}$ or Others. We consequently clustered these haplogroups following the classification suggested by MITOMAP and followed by another study assessing mitochondrial haplogroups and cardiovascular disease (CVD) $)^{14,15}$ into HV, JT, KU, IWX, and superHV/others.

\section{Outcomes}

In the OAI, a trained nurse measured weight and height with standardized methods. Weight was recorded without shoes, heavy jewelry, or wallets, and in lightweight clothing in $\mathrm{Kg}$. Height was measured without shoes, using the required breathing technique during each 
measurement. Two initial measurements were made, with the participant a step away from the stadiometer, then step back into the measurement position. If the difference between these two measurements was greater than $4 \mathrm{~mm}$, other two measurements were made. Height was recorded in $\mathrm{mm}$. BMI $\geq 30 \mathrm{~kg} / \mathrm{m}^{2}$ in agreement with the guidelines suggested by the Word Health Organization (WHO) ${ }^{16}$ and computed as weight/height ${ }^{2}$ These measures were repeated at each visit, i.e. V01, V03, V05, V06, V08, and V10.

\section{Covariates}

Obesity risk across mitochondrial haplogroups was examined after controlling for the following potential confounders (1) baseline BMI; (2) physical activity, using PACE ${ }^{17}$, which assesses 12 different physical activities (e.g. walking, housework). It is scored on a 0-400 scale and has been validated in older populations; (3) smoking habits (previous/current vs. never); (4) education ( degree vs. lower levels); (5) annual income ( $\geq \$ 50,000$ vs. $<\$ 50,000$ or missing data); (6) co-morbidities, quantified with the modified Charlson scale (higher scores indicate greater severity); and (7) total energy intake (in daily Kcal) assessed with a food frequency questionnaire. ${ }^{18}$

\section{Statistical analyses}

Means and standard deviations (continuous outcomes) and count percentage (categorical outcomes) were computed for mitochondrial haplogroups, with ANOVA and Pearson's chisquare used as corresponding inferential tests. Normality was assessed with the KolmogorovSmirnov test. We used Levene's test to assess equality of variance, with Welch's ANOVA used in the event of any violation of this assumption. Post-hoc analyses were run and Bonferroni adjustments were applied. The haplotype HV (the most common) was used as the reference group in all comparisons. 
Proportional hazards (Cox) regression was used to quantify the magnitude of the association of mitochondrial haplogroups and incident obesity, with an outcome of time to the onset of obesity or to the last observation made. All the analyses excluded individuals with obesity at baseline. Participant attrition due to death was censored at the date of death.

Factors associated with obesity at follow-up in the univariable analysis were included in the model. A VIF (variance inflation factor) of $>=2$ was used as a threshold for multicollinearity The baseline model was adjusted for age and sex, while the fully-adjusted model also controlled for baseline BMI, education, smoking, income, co-morbidity, physical activity and daily energy intake (see previous section for how these variables were categorized). . Hazard ratios (HRs) with 95\% confidence intervals (CIs) were used as the primary outcome statistic.

Analyses were conducted with SPSS 17.0, with using $\mathrm{p}<.05$ used to indicate significance. 


\section{RESULTS}

\section{Participants}

Haplogroup data was not available for 1,047 of 4,796 participantswith data from a further 126 excluded due to technical problems. Moreover, 1,167 people were already obese at baseline, whilst other 114 were lost at follow-up. Therefore, the final sample of the current study consisted of 2,342 eligible individuals (Figure 1).

\section{Baseline analyses}

The 2,342 participants included had a mean age of $62.0 \pm 9.5$ (45-79) years, with slightly more women $(=56.7 \%)$. All the participants included were Caucasian and the mean \pm SD BMI at baseline was $25.6 \pm 2.7 \mathrm{Kg} / \mathrm{m}^{2}$.

Participant baseline characteristics by mitochondrial haplogroup are reported in Table 1. The HV group was the most frequent haplotype $(\mathrm{n}=1,051 ;=44.9 \%)$ and was used as the reference in all the elaborations. Compared to the HV haplogroup, there were no significant differences in age, sex, physical activity level, smoking, comorbidity, or energy intake demonstrated across other haplogroups. Participants having IWX haplotype $(n=100)$ consisted of a significantly higher prevalence of highly educated people $(\mathrm{p}=0.02)$, but these participants were significantly richer $(\mathrm{p}=0.01)$ than the reference group (HV). Finally, the 142 having super HV/other mitochondrial haplotype showed significantly higher BMI values than people with HV haplotype (Table 1).

\section{Association between mitochondrial haplogroups and incident obesity}

After a median period of 8 years, 334 individuals (14.3\% of baseline population) developed obesity. 
As shown in Table 2, the incidence of obesity was higher in people with the haplotype IWX (31; 95\%CI: 20-48 per 1,000 persons-year) and others/superHV haplogroups (35; 95\%CI: 25 50 per 1,000 persons-year) compared to the reference group, HV (22; 95\% CI: $18-25$ per 1,000 persons-year) (Figure 1).

Using a Cox's regression analysis adjusted for nine demographic and clinical potential confounders and taking people with the HV haplotype as reference, the haplogroup IWX carried a significantly higher risk for obesity by about $48 \%(\mathrm{HR}=1.48 ; 95 \% \mathrm{CI}: 1.02-2.39)$

(Table 2).

\section{DISCUSSION}

To the best of our knowledge, the current study is the first longitudinal investigation of the relationship between mitochondrial haplogroups and obesity in a cohort of community dwelling adults. We found that over a follow-up period of 8 years, people who had an IWX haplotype $(n=100 ;=4.2 \%$ of the baseline population) were approximately $48 \%$ more likely to develop obesity compared to the most frequent haplotype (HV). This finding remained significant even when accounting for several potential confounders assessed at baseline which are known to influence the risk of obesity, such as physical activity and education status.

It is noteworthy that at baseline, participants with the IWX haplotype did not have any significant difference in potential risk factors for obesity, such as baseline BMI. However, after 8 years of follow-up, the IWX haplogroup carried a sizeable and consistent increase of nearly $50 \%$ in obesity incidence, suggesting an important role of these haplotypes for this condition. In contrast to previous literature regarding this topic, haplogroup $\mathrm{T}$ was not linked to obesity. However, in the most recent study regarding the possible association between mitochondrial haplogroups and obesity, the relationship between haplogroup $\mathrm{T}$ and juvenile obesity disappeared after adjustment 
for multiple testing. ${ }^{10}$ Several reasons can explain the differences between our study and those reporting a significant association between haplogroup $\mathrm{T}$ and obesity. First, we used a different categorization of mitochondrial haplogroups compared to the case control studies mentioned and consequently a different reference group. In particular, we grouped the group $\mathrm{T}$ with the $\mathrm{J}$ as suggested by MITOMAP and in agreement with other works suggesting that mitochondrial haplogroup $\mathrm{J}$ and $\mathrm{T}$ form a cluster with shared polymorphisms (e.g. mtDNA T4216C) and were formerly described as sister haplogroups with a common root. ${ }^{19}$ Second, the design of our study (longitudinal vs. case control) may lead to different results.

The precise reason why the IWX haplogroup is linked to obesity is unclear and clearly warrants investigation. One potential reason may be that participants of these haplogroups may have had lower energy expenditure and higher fat accumulation. In a study including 231 subjects suffering from AIDS, the ten people of haplogroup I exhibited higher baseline extremity fat compared to other haplogroups, whilst those in haplogroup W had the greatest increase in extremity fat over 96 months of follow-up. ${ }^{20}$ However, other studies are needed to confirm these findings coming from a special population.

Our findings are of importance since obesity is increasing worldwide. As recently as 2010, 3.4 million deaths were linked to obesity, as well as nearly $4 \%$ of disability-adjusted life-years worldwide. ${ }^{21}$ While there are clearly substantive environmental contributions to obesity, genetic components are also likely to contribute with heritability estimates of $40-70 \%$ suggested. ${ }^{22}$ Obesity is a multi-factorial disease due to an interaction between environmental and genetic factors. Among genetic factors, Genome-Wide Association Studies (GWAS) suggested that a core network module in humans and mice is possible. This network module is rich of genes involved in the inflammatory and immune response and probably play a role for obesity-related traits. ${ }^{23}$ For a long time, it was discussed if a person may become obese for a low energy expenditure or for a too high energy 
intake with diet. ${ }^{24}$ Low energy expenditure is traditionally seen as risk factor for obesity. In industrialized countries, it is common for half of a waking day sitting and the other half consisting of low volume physical activity. ${ }^{25}$ Recent studies suggest that the low energy expenditure resulting from this low level of activity may have a significant direct relationship with obesity. ${ }^{26}$ However, more recent studies proposed that high energy intake may have a more important role than low energy expenditure in determining obesity. ${ }^{27}$ The role of genetic factors in determining obesity is very conflicting and still debated. As shown by several studies ${ }^{28,29}$, most people probably have some genetic predisposition to obesity, but the movement from genetic predisposition to obesity itself requires a strong interaction with other environmental factors, such as diet and lifestyle. ${ }^{30}$ However, contrary to the literature regarding nuclear DNA, the literature regarding mitochondrial haplogroups is still limited, but could be of importance since the sequencing of the complete mtDNA genome is now readily available, and in the coming era of personalized medicine, these types of genetic discoveries may integrate these information into clinical practice, for tailored therapeutic intervention strategies. ${ }^{31}$

It is important to note that these findings should be treated cautiously. First, data were only available for Caucasians and thus cannot be automatically genralised to outside of this specific ethnic group. Second, the OAI study included only people with or at high risk of knee osteoarthritis, and so the same concerns about genralisability to wider population must apply. These limitations notwithstanding, the large cohort included at baseline, the control for a number of important confounders and the long-follow-up period, make an important contribution to our understanding of how genetic haplotype may confer a vulnerability to obesity.

In conclusion, in our study, mitochondrial haplogroups IWX carried a significant higher risk of obesity compared to the most frequent haplogroups, HV. Future longitudinal studies in general population are however needed to confirm/refute our findings. 


\section{ACKNOWLEDGMENTS}

Funding sources: The OAI is funded by the National Institute of Health. Private funding partners include Merck Research Laboratories; Novartis Pharmaceuticals Corporation, GlaxoSmithKline; and Pfizer, Inc. Private sector funding for the OAI is managed by the Foundation for the National Institutes of Health. This manuscript was prepared using an OAI public use data set and does not necessarily reflect the opinions or views of the OAI investigators, the NIH, or the private funding partners.

Role of founding source: Funding sources had no role in study design, data treatment, or report writing.

Conflict of interest: None. 


\section{REFERENCES}

1. Wang Y, Brinton RD. Triad of Risk for Late Onset Alzheimer's: Mitochondrial Haplotype, APOE Genotype and Chromosomal Sex. Frontiers in aging neuroscience 2016; 8: 232.

2. Copeland WC, Longley MJ. Mitochondrial genome maintenance in health and disease. DNA repair 2014; 19: 190-8.

3. Wallace DC, Chalkia D. Mitochondrial DNA genetics and the heteroplasmy conundrum in evolution and disease. Cold Spring Harbor perspectives in biology 2013; 5(11): a021220.

4. Picard M, Wallace DC, Burelle Y. The rise of mitochondria in medicine. Mitochondrion 2016; 30: 105-16.

5. Mishmar D, Ruiz-Pesini E, Golik P, Macaulay V, Clark AG, Hosseini S et al. Natural selection shaped regional mtDNA variation in humans. Proceedings of the National Academy of Sciences of the United States of America 2003; 100(1): 171-6.

6. Veronese N, Stubbs B, Solmi M, Vaona A, Demurtas J, Carvalho AF et al. Mitochondrial genetic haplogroups and depressive symptoms: A large study among people in North America. J Affect Disord 2017; 217: 55-59.

7. Wortmann SB, Zweers-van Essen H, Rodenburg RJ, van den Heuvel LP, de Vries MC, Rasmussen-Conrad E et al. Mitochondrial energy production correlates with the age-related BMI. Pediatric research 2009; 65(1): 103-8.

8. Yang TL, Guo Y, Shen H, Lei SF, Liu YJ, Li J et al. Genetic association study of common mitochondrial variants on body fat mass. PloS one 2011; 6(6): e21595.

9. Nardelli C, Labruna G, Liguori R, Mazzaccara C, Ferrigno M, Capobianco V et al. Haplogroup $\mathrm{T}$ is an obesity risk factor: mitochondrial DNA haplotyping in a morbid obese population from southern Italy. BioMed research international 2013; 2013: 631082.

10. Ebner S, Mangge H, Langhof H, Halle M, Siegrist M, Aigner E et al. Mitochondrial Haplogroup T Is Associated with Obesity in Austrian Juveniles and Adults. PloS one 2015; 10(8): e 0135622.

11. Grant SF, Glessner JT, Bradfield JP, Zhao J, Tirone JE, Berkowitz RI et al. Lack of relationship between mitochondrial heteroplasmy or variation and childhood obesity. International journal of obesity (2005) 2012; 36(1): 80-3.

12. Rego-Perez I, Fernandez-Moreno M, Fernandez-Lopez C, Arenas J, Blanco FJ. Mitochondrial DNA haplogroups: role in the prevalence and severity of knee osteoarthritis. Arthritis and rheumatism 2008; 58(8): 2387-96.

13. Torroni A, Huoponen $\mathrm{K}$, Francalacci $\mathrm{P}$, Petrozzi M, Morelli L, Scozzari $\mathrm{R}$ et al. Classification of European mtDNAs from an analysis of three European populations. Genetics 1996; 144(4): 1835-50.

14. MITOMAP. MITOMAP: a human mitochondrial genome database In, 2017. 
15. Fernandez-Caggiano M, Barallobre-Barreiro J, Rego-Perez I, Crespo-Leiro MG, Paniagua MJ, Grille $\mathrm{Z}$ et al. Mitochondrial haplogroups $\mathrm{H}$ and $\mathrm{J}$ : risk and protective factors for ischemic cardiomyopathy. PloS one 2012; 7(8): e44128.

16. Obesity: preventing and managing the global epidemic. Report of a WHO consultation. World Health Organization technical report series 2000; 894: i-xii, 1-253.

17. Washburn RA, McAuley E, Katula J, Mihalko SL, Boileau RA. The physical activity scale for the elderly (PASE): evidence for validity. Journal of clinical epidemiology 1999; 52(7): 643-51.

18. Veronese N, Stubbs B, Noale M, Solmi M, Vaona A, demurtas J et al. Fried potato consumption is associated with elevated mortality: an 8-year longitudinal cohort study. The American journal of clinical nutrition 2017.

19. Ruiz-Pesini E, Mishmar D, Brandon M, Procaccio V, Wallace DC. Effects of purifying and adaptive selection on regional variation in human mtDNA. Science (New York, N.Y.) 2004; 303(5655): 223-6.

20. Hulgan T, Haubrich R, Riddler SA, Tebas P, Ritchie MD, McComsey GA et al. European Mitochondrial DNA Haplogroups and Metabolic Changes during Antiretroviral Therapy in AIDS Clinical Trials Group Study A5142. AIDS (London, England) 2011; 25(1): 37-47.

21. Ng M, Fleming T, Robinson M, Thomson B, Graetz N, Margono C et al. Global, regional, and national prevalence of overweight and obesity in children and adults during 1980\&\#x2013;2013: a systematic analysis for the Global Burden of Disease Study 2013. The Lancet 2014; 384(9945): 766-781.

22. Herrera BM, Lindgren CM. The Genetics of Obesity. Current Diabetes Reports 2010; 10(6): 498-505.

23. Emilsson V, Thorleifsson G, Zhang B, Leonardson AS, Zink F, Zhu J et al. Genetics of gene expression and its effect on disease. Nature 2008; 452(7186): 423-8.

24. Hill JO, Wyatt HR, Peters JC. Energy Balance and Obesity. Circulation 2012; 126(1): 126132.

25. Hamilton MT, Hamilton DG, Zderic TW. Role of low energy expenditure and sitting in obesity, metabolic syndrome, type 2 diabetes, and cardiovascular disease. Diabetes 2007; 56(11): 2655-67.

26. Barnes AS. Obesity and Sedentary Lifestyles: Risk for Cardiovascular Disease in Women. Texas Heart Institute Journal 2012; 39(2): 224-227.

27. Caudwell P, Gibbons C, Finlayson G, Näslund E, Blundell J. Physical Activity, Energy Intake, and Obesity: The Links Between Exercise and Appetite. Current Obesity Reports 2013; 2(2): 185-190.

28. Bradfield JP, Taal HR, Timpson NJ, Scherag A, Lecoeur C, Warrington NM et al. A genome-wide association meta-analysis identifies new childhood obesity loci. Nature genetics 2012; 44(5): 526-531. 
29. Locke AE, Kahali B, Berndt SI, Justice AE, Pers TH, Day FR et al. Genetic studies of body mass index yield new insights for obesity biology. Nature 2015; 518(7538): 197-206.

30. Hu F. Obesity epidemiology, Oxford University Press, 2008.

31. Meschia JF, Ross OA. Does mitochondrial DNA have a protective role in stroke? The Lancet. Neurology 2010; 9(5): 453-4. 


\section{FIGURE LEGENDS}

Figure 1. Flow chart of the study

Patients enrolled in the OAI study: $\mathbf{4 , 7 9 6}$

Patients excluded and reasons:

1,047: no data regarding mitochondrial haplogroups

126: technical problems

1,167: already obese at baseline

114: lost at follow-up

Patients included in the present study: $\mathbf{2 , 3 4 2}$ 
Table 1. Baseline characteristics by mitochondrial haplogroups.

\begin{tabular}{lccccc}
\hline \multicolumn{1}{c}{ Variable } & HV & JT & KU & IWX & SuperHV/ \\
& $(\mathbf{n = 1 0 5 1 )}$ & $(\mathbf{n = 4 6 7 )}$ & $(\mathbf{n = 5 8 2})$ & $(\mathbf{n = 1 0 0})$ & others \\
& & & & & $(\mathbf{n}=\mathbf{1 4 2})$ \\
\hline Age (years) & $61.7(9.6)$ & $62.1(9.6)$ & $62.8(9.2)$ & $61.7(9.9)$ & $61.8(9.2)$ \\
Females (\%) & 58.6 & 57.4 & 53.4 & 51.0 & 57.7 \\
PASE (points) & $169(92)$ & $168(101)$ & $172(105)$ & $172(77)$ & $155(81)$ \\
Smoking (\%) & 43.9 & 44.5 & 45.9 & 45.0 & 45.8 \\
Degree (\%) & 36.5 & 35.5 & 36.8 & $42.0^{*}$ & 30.3 \\
Yearly income (<50,000 \$) & 67.6 & 68.5 & 66.7 & $56.0^{*}$ & 59.2 \\
Charlson co-morbidity score & $0.3(0.7)$ & $0.3(0.7)$ & $0.3(0.7)$ & $0.4(1.0)$ & $0.4(0.9)$ \\
Energy intake (kcal/day) & $1380(511)$ & $1343(513)$ & $1344(537)$ & $1404(563)$ & $1410(511)$ \\
BMI (Kg/m $\left.{ }^{2}\right)$ & $25.6(2.7)$ & $25.5(2.79$ & $25.4(2.7)$ & $25.6(2.9)$ & $26.3(2.7)^{*}$
\end{tabular}

\section{Notes:}

In all the comparisons, the group HV was taken as reference.

${ }^{* * *}: \mathrm{p}<0.0001 ;{ }^{* *}: \mathrm{p}<0.001 ;{ }^{*}: \mathrm{p}<0.05$. The Bonferroni’s correction was applied for continuous measures.

Numbers are mean values (and standard deviations) or percentages, as appropriate.

Abbreviations: BMI: body mass index; PASE: physical activity scale for the elderly. 
Table 2. Association between mitochondrial haplogroups and incident obesity.

\begin{tabular}{|c|c|c|c|c|c|}
\hline & Incidence & Basic adjusted & & Fully adjusted & \\
\hline & $(95 \% \mathrm{CI})$ & $\mathbf{H R}^{*}$ & $P$ value & $\mathbf{H R}^{* * *}$ & $P$ value \\
\hline & per 1,000 persons-year & $(95 \% \mathrm{CI})$ & & $(95 \% \mathrm{CI})$ & \\
\hline HV & $22(18-25)$ & $1[\mathrm{re}$ & & $1[\mathrm{re}$ & \\
\hline JT & $19(15-25)$ & $0.96(0.71-1.299$ & 0.77 & $1.05(0.78-1.42)$ & 0.74 \\
\hline KU & $19(15-24)$ & $0.92(0.70-1.22)$ & 0.57 & $1.03(0.77-1.37)$ & 0.85 \\
\hline IWX & $31(20-48)$ & $1.52(1.08-2.45)$ & 0.02 & $1.48(1.02-2.39)$ & 0.01 \\
\hline Others/ SuperHV & $35(25-50)$ & $1.62(1.09-2.41)$ & 0.02 & $1.14(0.76-1.71)$ & 0.54 \\
\hline
\end{tabular}

\section{Notes:}

* Basic adjusted model includes age (as continuous) and sex.

${ }^{* *}$ Fully-adjusted model included as covariates: body mass index (as continuous) at baseline; education (degree vs. others); smoking habits (current and previous vs. others); yearly income (categorized as $\geq$ or $<50,000 \$$ and missing data); Charlson co-morbidity index (as continuous); Physical Activity Scale for Elderly score (as continuous); daily energy intake (as continuous).

Abbreviations: CI: confidence intervals; HR: hazard ratio. 